\title{
Apical hypertrophic cardiomyopathy with apical aneurysm
}

\author{
Jon-David Schwalm MD FRCPC, Mayraj Ahmad MD FRCPC, Amin Mulji MD FRCPC
}

\begin{abstract}
A pical hypertrophic cardiomyopathy (AHCM), first A described by Sakamoto et al (1), is typically characterized by 'giant negative $T$ waves' (larger than $10 \mathrm{~mm}$ ), particularly in the precordial leads, asymmetrical left ventricular (LV) apical hypertrophy on echocardiogram and spade-shaped LV configuration on left ventriculography (2).

An asymptomatic 55-year-old woman had repolarization abnormalities noted on a routine preoperative electrocardiogram (Figure 1A). The initial echocardiogram was normal, with no evidence of LV hypertrophy or segmental wall motion abnormalities. Subsequent investigations, including coronary catheterization and cardiac magnetic resonance imaging, led to the diagnosis of AHCM with LV apical aneurysm (Figures $1 \mathrm{~B}$ and $1 \mathrm{C}$ ).

The present case illustrates that although the electrocardiogram and echocardiogram are commonly used in clinical practice, these tests may not be sufficient to exclude the diagnosis of AHCM. Further investigations, including cardiac magnetic resonance imaging, are essential to the diagnosis of this rare condition $(3,4)$. The second point illustrated by the present case is that patients with AHCM are at risk of developing an aneurysm at the LV apex, which can potentially lead to increased incidence of embolic complications, arrhythmias and congestive heart failure (5).
\end{abstract}

\section{REFERENCES}

1. Sakamoto T, Tei C, Murayama M, Ichiyasu H, Hada Y. Giant T wave inversion as a manifestation of asymmetrical apical hypertrophy (AAH) of the left ventricle. Echocardiographic and ultrasono-cardiotomographic study. Jpn Heart J 1976;17:611-29.

2. Yamaguchi H, Ishimura T, Nishiyama S, et al. Hypertrophic nonobstructive cardiomyopathy with giant negative $\mathrm{T}$ waves (apical hypertrophy): Ventriculographic and echocardiographic features in 30 patients. Am J Cardiol 1979;44:401-12.

3. Pons-Lladó G, Carreras F, Borrás X, Palmer J, Llauger J, Bayés de Luna A. Comparison of morphologic assessment of hypertrophic cardiomyopathy by magnetic resonance versus echocardiographic imaging. Am J Cardiol 1997;79:1651-6.
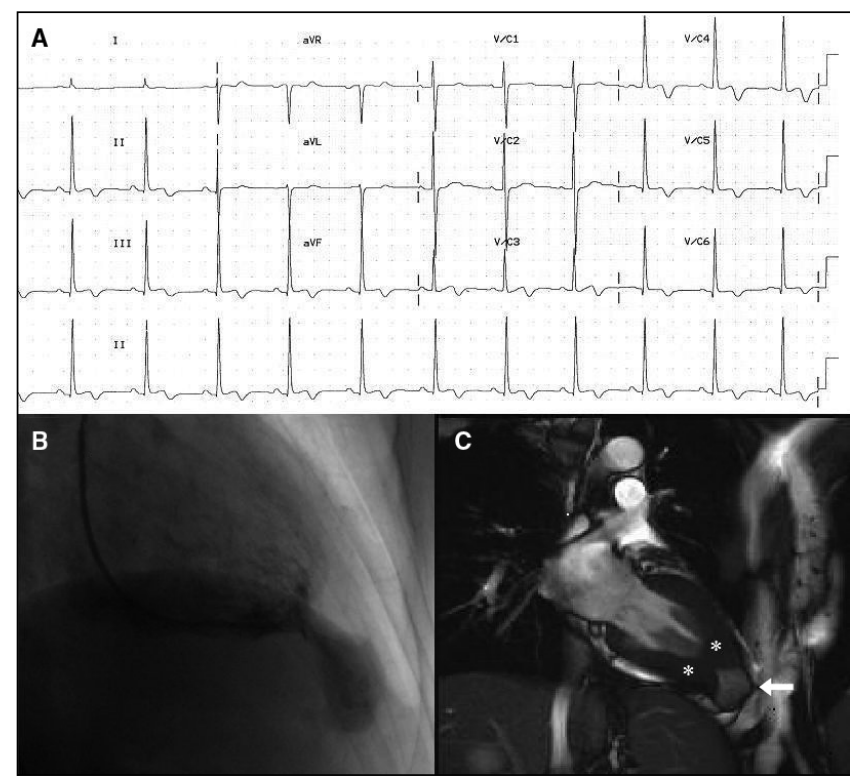

Figure 1) A Resting 12-lead electrocardiogram demonstrating voltage criteria for left ventricular hypertrophy and negative $T$ waves of $2 \mathrm{~mm}$ to $4 \mathrm{~mm}$ in leads II, III, aVF and $V_{3}$ to $V_{6}$. B Left ventricular angiogram demonstrating apical aneurysm. $\mathrm{C}$ Left ventricular long-axis two-chamber cine cardiac magnetic resonance image showing thinwalled apical aneurysm (arrow) and apical hypertrophy (asterisks)

4. Moon JC, Fisher NG, McKenna WJ, Pennell DJ. Detection of apical hypertrophic cardiomyopathy by cardiovascular magnetic resonance in patients with non-diagnostic echocardiography. Heart 2004;90:645-9.

5. Matsubara K, Nakamura T, Kuribayashi T, Azuma A, Nakagawa M. Sustained cavity obliteration and apical aneurysm formation in apical hypertrophic cardiomyopathy. J Am Coll Cardiol 2003;42:288-95. (Erratum in 2003;42:1338).

Department of Medicine, Division of Cardiology, McMaster University, Hamilton General Hospital Site, Hamilton, Ontario

Correspondence: Dr Jon-David Schwalm, Hamilton Health Sciences, McMaster University, 237 Barton Street East, Hamilton, Ontario L8L 2 X2. Telephone 905-317-0135, fax 905-527-1914, e-mail schwalj@mcmaster.ca

Received for publication July 19, 2006. Accepted July 24, 2006 